\title{
Multiple extraspinal recurrences of ependymoma 13 years after spinal cordectomy: case report
}

\author{
Wittstatt Alexandra Whitaker-Lea, MD, ${ }^{1}$ Robert Scott Graham, MD, ${ }^{1}$ Jan Paul Muizelaar, MD, PhD, ${ }^{2}$ \\ and Hope T. Richard, MD, $\mathrm{PhD}^{3}$
}

\begin{abstract}
Departments of ${ }^{1}$ Neurological Surgery and ${ }^{3}$ Pathology, Virginia Commonwealth University Health System, Richmond, Virginia; and ${ }^{2}$ Department of Neurological Surgery, Marshall University, Huntington, West Virginia
\end{abstract}

\begin{abstract}
Ependymomas of the spinal cord remain confined to the CNS and vary in presentation, depending on WHO grade. Higher-grade lesions usually cannot be surgically removed due to their infiltrative growth pattern. Spinal cordectomy has been proposed as a rescue treatment to improve survival in patients with high-grade as well as recurrent lesions. This report details an instructive and unique case of long-term follow-up of a patient who underwent cordectomy from T-4 through S-5 for what was initially diagnosed as a high-grade glial neoplasm of the spinal cord in 1993. The patient lived symptom free for 13 years after spinal cord resection and then presented with numerous bilateral extraspinal (intraabdominal and intrathoracic) tumors, which eventually led to her death 15 years after the cordectomy. In this case, spinal cordectomy was effective in preventing the ascending spread of the neoplasm, but ultimately not effective in preventing recurrence in the plicated distal dural sac.
\end{abstract}

https://thejns.org/doi/abs/10.3171/2017.12.SPINE17848

KEYWORDS ependymoma; spinal cordectomy; surgery; oncology

$\mathrm{S}$ PINAL cord ependymomas are tumors exclusive to the CNS that arise from the ependymal cells that line the central canal of the spinal cord. Spinal cord ependymomas account for $2 \%-6 \%$ of all CNS tumors, are the most common primary intramedullary spinal cord tumor in adults, and most commonly present in the 3rd to 4th decades of life. ${ }^{6,17,18}$ Symptoms are generally progressive over several years for the more benign variants, but symptoms progress over a much shorter period for the malignant variants. ${ }^{17}$ There are several histological subtypes defined by the WHO that are further delineated as grades I-III. ${ }^{12}$ Primary spinal anaplastic ependymomas are extremely rare and account for only $7 \%$ of all spinal ependymomas. ${ }^{4,7}$

The primary treatment for a spinal cord ependymoma is resection, and, although not without risks, gross-total resection is considered the greatest prognostic factor for overall survival. ${ }^{1,6,7,9,10,13-17,20,21}$ Radiation therapy, generally
45-54 Gy, has also been reported to prolong progressionfree survival in patients who are poor surgical candidates, patients with residual tumor, tumors with anaplastic features, or tumors resected in a piecemeal fashion. ${ }^{3,6-8,15,19}$ Chamberlain also advocated for the use of etoposide as salvage chemotherapy in patients with recurrent ependymomas. ${ }^{5}$ Spinal cordectomy is a rare procedure that is used to control disease spread in patients in whom other options have been exhausted or are not feasible. ${ }^{11}$ This case report describes the delayed extraspinal recurrence of an ependymoma 13 years after spinal cordectomy.

\section{Case Report}

History, Physical Examination, and Initial Imaging

In 1993, a 40-year-old female patient had a history of longstanding back pain that had worsened over the month 

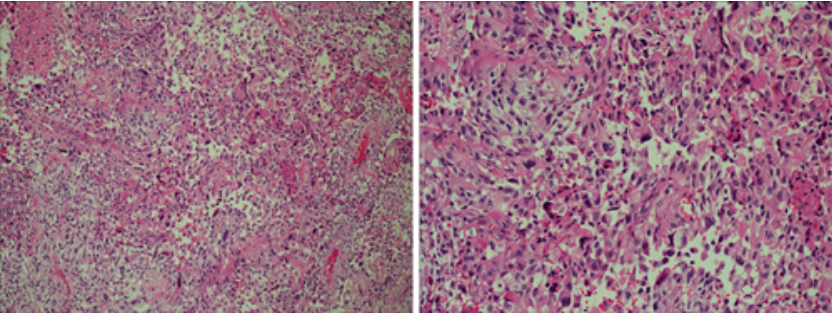

FIG. 1. Low-power (left) and high-power (right) views of an H \& Estained section revealing bizarre anaplastic cells with focal areas of necrosis. Original magnification $\times 40$ (left); $\times 100$ (right). Figure is available in color online only.

prior to presentation. She experienced progressive paraparesis of the lower extremities for 1 week and presented to the emergency department with acute worsening of back pain and lower extremity weakness after a fall earlier that day. The patient also reported constipation and urinary retention, particularly on the day of presentation.

Physical examination was remarkable for $4 / 5$ strength in the bilateral hip flexors, diminished sensation in the T11-L2 dermatomes, and absent proprioception and deep tendon reflexes in the lower extremities. A straight catheter was placed, and more than $1 \mathrm{~L}$ of urine was drained.

MRI of the thoracic and lumbar spine with and without contrast showed an intramedullary enhancing mass from T-10 to L-1. There was expansion of the spinal cord to the level of T-1. Of note, there was no evidence of metastasis on initial imaging. MRI of the cervical spine without contrast was also performed on presentation and the findings were significant only for a right-sided C6-7 lateral disc herniation. MRI of the brain was not performed.

\section{Operative Intervention and Pathologic Findings}

One day after presentation to the emergency department, the patient underwent T-11 and T-12 laminectomies and biopsy of the lesion. Histological examination yielded diagnosis of anaplastic astrocytoma (Fig. 1). Cells were positive for GFAP and negative for HMB 45 and cytokeratin, confirming glial cell origin, and the neuropathologist suggested that the lesion was a glioblastoma (GBM).

The patient was discharged to a rehabilitation facility with the intention to return for definitive intervention. The patient's condition continued to decline, and, at the time of readmission from rehabilitation, she was noted to have $2 / 5$ strength in her bilateral lower extremities as well as a

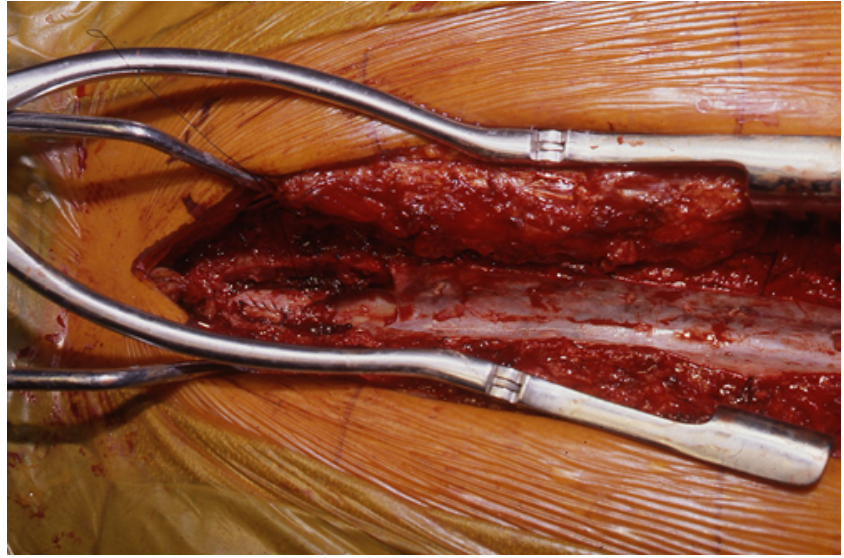

FIG. 3. Intraoperative photograph obtained after the spinal cordectomy, showing closure of the distal dural sac. Figure is available in color online only.

T-10 sensory level. With the belief that the tumor was a GBM, and no literature at that time reporting any survival beyond 3 months after diagnosis, the surgeon (J.P.M.) discussed with the patient and her husband that radical cordectomy might offer the only hope for long-term survival. The patient agreed, but wanted to keep sensation in her nipples, hence the choice of the entrance of the T-5 nerve root into the cord as the upper limit of the cordectomy. Fifteen days after the initial biopsy, T3-L3 laminectomies and cordectomy distal to T-4 was performed (Fig. 2). Bipolar cauterization of the nerve roots and corresponding radicular arteries was performed, followed by transection with microscissors. Dentate ligaments were cut using microscissors. In this specimen, neoplastic cells were noted in the conus, but the margins were negative. No evidence of subarachnoid tumor spread was seen. The pathologist confirmed the diagnosis of GBM. The dural sac was sewn closed at the level of the cord resection (Fig. 3).

\section{Postoperative Course and Re-Presentation}

Postoperatively, the patient had no motor function in her lower extremities, a T-4 sensory level, and complete loss of bowel and bladder function. She recovered without complication and was transferred back to rehabilitation on postoperative day 5 . The decision was made to not perform postoperative MRI, as the surgeon was confident that the tumor had been removed in its entirety and felt that imaging would not change management. Additionally, no

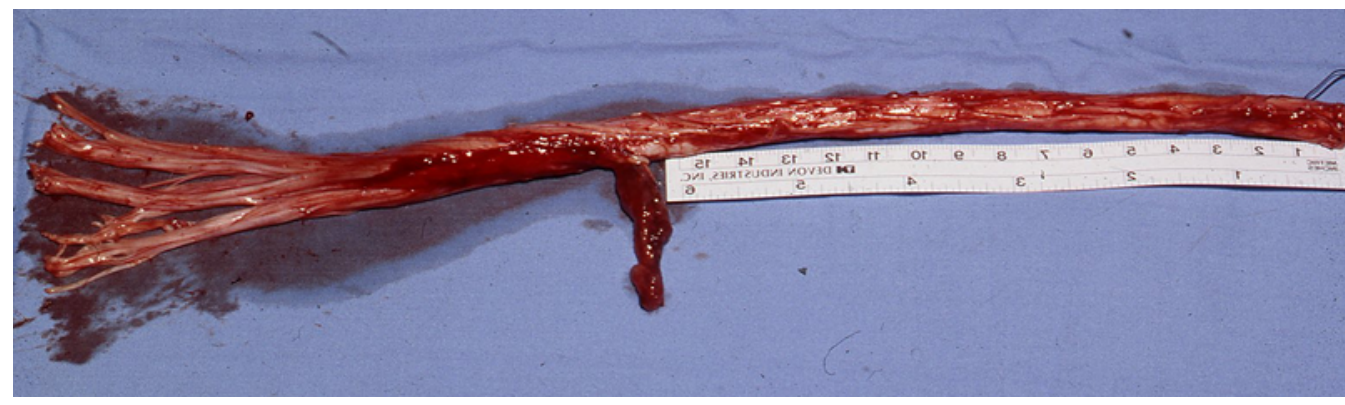

FIG. 2. Intraoperative photograph of the spinal cord removed during the cordectomy. Figure is available in color online only. 

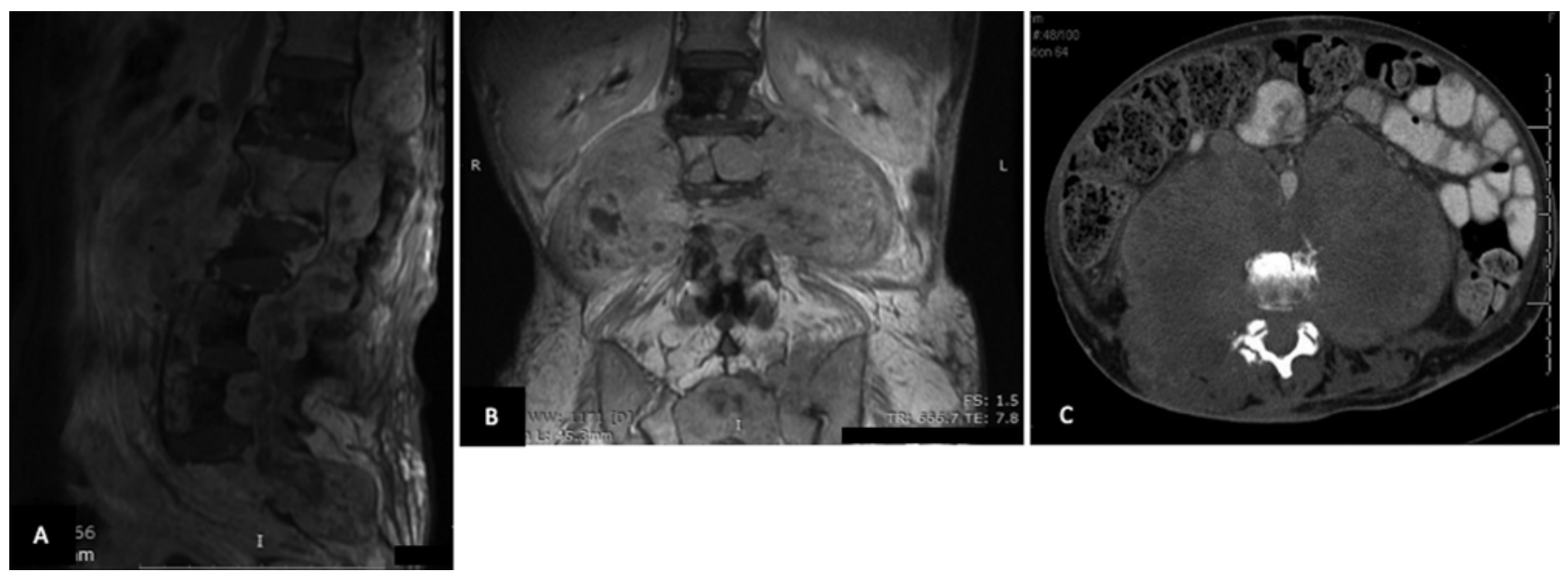

FIG. 4. Sagittal (A) and coronal (B) MR images without contrast obtained around the time of re-presentation. Axial CT scan obtained 1 month prior to the patient's death (C).

adjuvant therapy was administered, given the complete resection of the tumor. After discharge from rehabilitation, the patient had one follow-up appointment approximately 5 months postoperatively. At that time, she reported midback pain and an unusual sensation of electric shocks in her legs. The incision was well healed. After this appointment, the patient stayed in telephone contact with her surgeon for a number of years, but was eventually lost to follow-up. It was not until 13 years later that the patient again presented to the neurosurgery service.

The patient re-presented to an outside hospital after 6 months of abdominal pain and lower-extremity edema. MRI revealed tumor recurrence at every neural foramen and within the retroperitoneal space (Fig. 4). Metastasis to the lung and liver, as well as a retrocardiac mass, was present. However, of note, at time of re-presentation no tumor was present within the CNS. CT-guided biopsy of the retroperitoneal portion of the mass performed at an outside hospital revealed a highly atypical, hypocellular

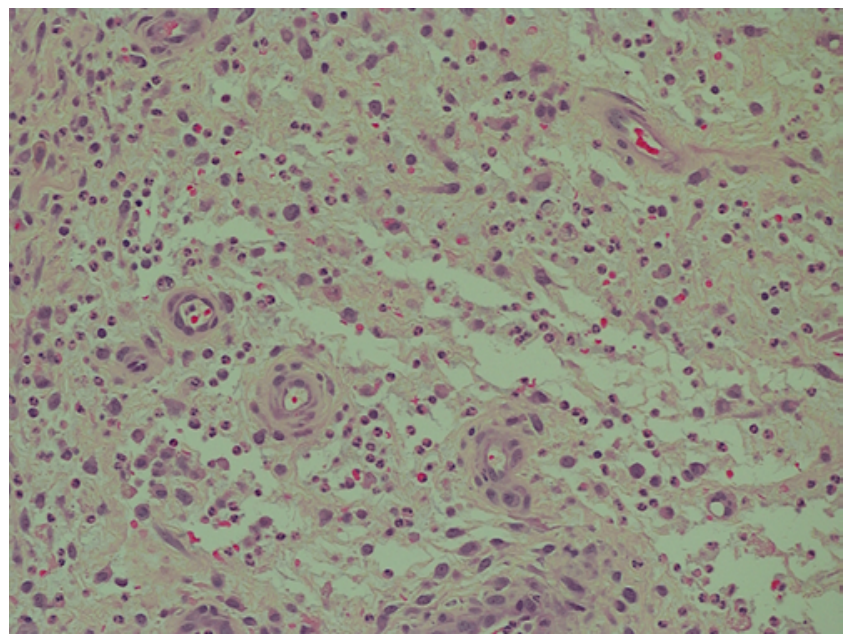

FIG. 5. High-power H \& E-stained section obtained during the CT-guided biopsy, revealing reactive cells. Original magnification $\times 200$. Figure is available in color online only. stoma-rich neoplasm (Fig. 5). Four separate pathologists at an outside hospital reviewed the biopsy results without coming to a uniform consensus. After the sample was reviewed at our institution, it was felt to be more consistent with the diagnosis of anaplastic ependymoma as opposed to the initial diagnosis of GBM. This change in diagnosis was likely due to the pattern of epithelial membrane antigen (EMA) staining. The patient was seen at our neurosurgery clinic approximately 11 months after symptoms recurred. Due to extensive involvement of spinal, paraspinal, and intraabdominal compartments, the patient was referred to neurooncology. She completed 3 rounds of temozolomide, with an initial positive response. However, her course was later complicated by seizures and intracranial metastasis requiring multiple hospital admissions. In July 2007, at age 55 years, the patient died.

Recently, the original specimen from the cordectomy in 1993 was restained. The pathology was most consistent with clear cell ependymoma, although the papillary subtype also remains in the differential diagnosis (Fig. 6). The specimen was also stained for EMA, GFAP, and Ki67 (Fig. 7). EMA staining showed a perinuclear pattern that was also consistent with the diagnosis of ependymoma, which is in contrast to the focal weak staining typically seen with a GBM.

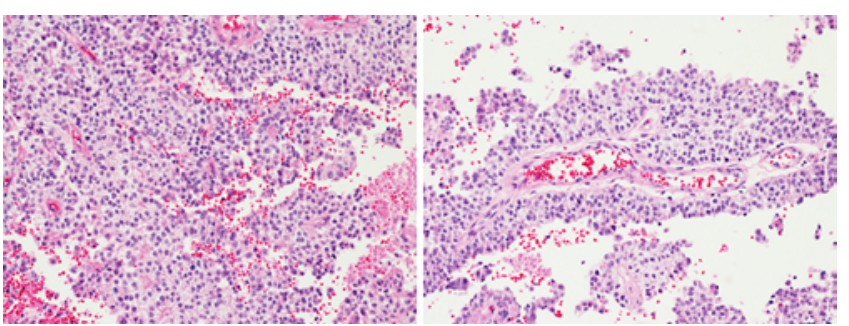

FIG. 6. Repeat H \& E-stained sections at high power obtained during the patient's initial biopsy, showing multiple pseudorosettes (left) and a single perivascular pseudorosette (right), consistent with the diagnosis of ependymoma. Original magnification $\times 100$ (left); $\times 200$ (right). Figure is available in color online only. 


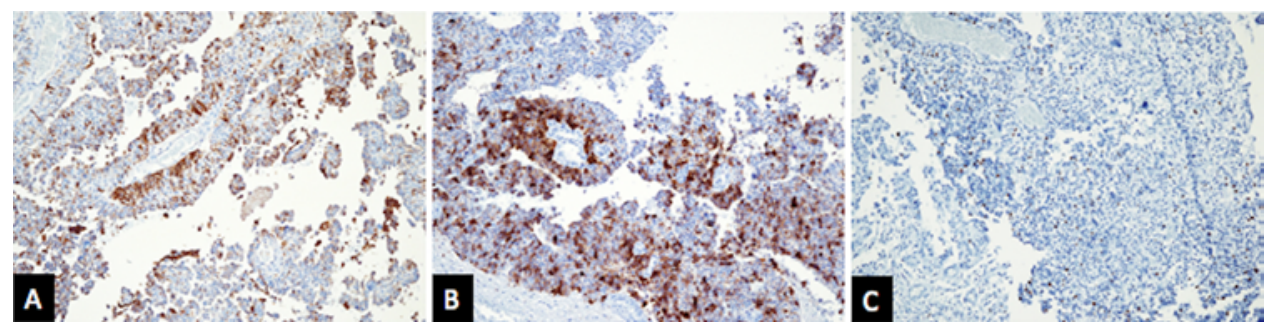

FIG. 7. GFAP (A), EMA (B), and Ki-67 (C) stains of the original resection, confirming the diagnosis of ependymoma. Figure is available in color online only.

\section{Discussion}

About $70 \%$ of spinal cord ependymoma recurrences are local, and most occur within 7 years. ${ }^{2}$ Alshaya et al. reported a case of delayed recurrence of a low-grade ependymoma and emphasized that there had only been 3 cases of delayed (12-19 years) metastasis of low-grade lesions at the time of publication. ${ }^{2}$

The decision to perform a cordectomy on initial presentation of our patient was based on the impression that the lesion was a GBM. This case report highlights the importance of staining for EMA when differentiating between GBM and ependymoma. Although the patient may have initially been misdiagnosed, it is very likely that she would have become paraplegic and without bowel and bladder control soon after initial presentation, given the rapid progression of her symptoms. Following gross-total resection, the average survival of patients with anaplastic ependymoma is 12.5 years, and with cordectomy as a salvage therapy, the longest documented survival after cordectomy for any grade ependymoma is 188 months. ${ }^{1,11}$ Our patient survived for 15 years, almost matching the longest documented survival.

In a literature review by Konar et al.in which the authors discussed the indications and outcomes after cordectomy, only 16 cases of spinal cordectomy for tumor were reported, of which only 3 were specifically for ependymomas. ${ }^{11}$ In these 3 patients, 1 did not have follow-up, and 2 patients were observed for 156 months and 188 months. Neither observed patient had recurrence. Delayed cerebrospinal fluid dissemination is a risk when performing cordectomy for malignant spinal cord neoplasm, but, to the best of our knowledge, there has not been a report of recurrence in the distal plicated sac after cordectomy for malignant spinal cord neoplasm. We speculate that the recurrence could have been a result of several factors. Although nerve roots and radicular arteries were cauterized and transected, it is possible that there were tumor cells distal to where the nerve was transected at the time of the cordectomy. Today, patients with both GBM and ependymoma of the spinal cord often undergo adjuvant therapy, including radiation therapy, chemotherapy, or both. Despite that there was no evidence at the time of resection that residual tumor was present, adjuvant therapy may have prevented recurrence in this patient.

In this case report, we present one of the earliest documented cases of spinal cordectomy that was used to treat a spinal cord ependymoma with an unusual delayed recurrence after 13 years. Undoubtedly, the spinal cordec- tomy in this case led to improved survival. However, the pattern of recurrence from the distal plicated dural sac following cordectomy has not been reported and highlights a potential therapeutic limitation of spinal cord resection.

\section{References}

1. Abdullah KG, Lubelski D, Miller J, Steinmetz MP, Shin JH, Krishnaney A, et al: Progression free survival and functional outcome after surgical resection of intramedullary ependymomas. J Clin Neurosci 22:1933-1937, 2015

2. Alshaya W, Mehta V, Wilson BA, Chafe S, Aronyk KE, Lu JQ: Low-grade ependymoma with late metastasis: autopsy case study and literature review. Childs Nerv Syst 31:15651572,2015

3. Celano E, Salehani A, Malcolm JG, Reinertsen E, Hadjipanayis CG: Spinal cord ependymoma: a review of the literature and case series of ten patients. J Neurooncol 128:377386, 2016

4. Cerase A, Venturi C, Oliveri G, De Falco D, Miracco C: Intradural extramedullary spinal anaplastic ependymoma. Case illustration. J Neurosurg Spine 5:476, 2006

5. Chamberlain MC: Salvage chemotherapy for recurrent spinal cord ependymona. Cancer 95:997-1002, 2002

6. Chang UK, Choe WJ, Chung SK, Chung CK, Kim HJ: Surgical outcome and prognostic factors of spinal intramedullary ependymomas in adults. J Neurooncol 57:133-139, 2002

7. Chen P, Sui M, Ye J, Wan Z, Chen F, Luo C: An integrative analysis of treatment, outcomes and prognostic factors for primary spinal anaplastic ependymomas. J Clin Neurosci 22:976-980, 2015

8. Gomez DR, Missett BT, Wara WM, Lamborn KR, Prados $\mathrm{MD}$, Chang S, et al: High failure rate in spinal ependymomas with long-term follow-up. Neuro Oncol 7:254-259, 2005

9. Keil VC, Schmitt AJ, Martin SC, Cadoux-Hudson TAD, Pereira EAC: Optimising treatment strategies in spinal ependymoma based on 20 years of experience at a single centre. J Clin Neurosci 29:52-58, 2016

10. Klekamp J: Spinal ependymomas. Part 1: Intramedullary ependymomas. Neurosurg Focus 39(2):E6, 2015

11. Konar SK, Maiti TK, Bir SC, Nanda A: Spinal cordectomy: a new hope for morbid spinal conditions. Clin Neurol Neurosurg 152:5-11, 2017

12. Louis DN, Perry A, Reifenberger G, von Deimling A, Figarella-Branger D, Cavenee WK, et al: The 2016 World Health Organization Classification of Tumors of the Central Nervous System: a summary. Acta Neuropathol 131:803-820, 2016

13. Montano N, Papacci F, Trevisi G, Fernandez E: Factors affecting functional outcome in patients with intramedullary spinal cord tumors: results from a literature analysis. Acta Neurol Belg 117:277-282, 2017

14. Nagasawa DT, Smith ZA, Cremer N, Fong C, Lu DC, Yang I: 
Complications associated with the treatment for spinal ependymomas. Neurosurg Focus 31(4):E13, 2011

15. Oh MC, Ivan ME, Sun MZ, Kaur G, Safaee M, Kim JM, et al: Adjuvant radiotherapy delays recurrence following subtotal resection of spinal cord ependymomas. Neuro Oncol 15:208-215, 2013

16. Oh MC, Kim JM, Kaur G, Safaee M, Sun MZ, Singh A, et al: Prognosis by tumor location in adults with spinal ependymomas. J Neurosurg Spine 18:226-235, 2013

17. Samartzis D, Gillis CC, Shih P, O'Toole JE, Fessler RG: Intramedullary spinal cord tumors: part I-epidemiology, pathophysiology, and diagnosis. Global Spine J 5:425-435, 2015

18. Tobin MK, Geraghty JR, Engelhard HH, Linninger AA, Mehta AI: Intramedullary spinal cord tumors: a review of current and future treatment strategies. Neurosurg Focus 39(2):E14, 2015

19. Volpp PB, Han K, Kagan AR, Tome M: Outcomes in treatment for intradural spinal cord ependymomas. Int J Radiat Oncol Biol Phys 69:1199-1204, 2007

20. Wu J, Armstrong TS, Gilbert MR: Biology and management of ependymomas. Neuro Oncol 18:902-913, 2016

21. Wu Z, Iwanami A, Yasuda A, Mikami S, Toyama Y, Nakamura M: Intramedullary cervicothoracic subependymoma: report of three cases and review of the literature. J Orthop Sci 20:927-934, 2015

\section{Disclosures}

The authors report no conflict of interest concerning the materials or methods used in this study or the findings specified in this paper.

\section{Author Contributions}

Conception and design: Whitaker-Lea, Graham, Muizelaar. Acquisition of data: Whitaker-Lea. Analysis and interpretation of data: Whitaker-Lea, Richard. Drafting the article: Whitaker-Lea, Graham, Muizelaar. Critically revising the article: Whitaker-Lea, Graham, Muizelaar. Reviewed submitted version of manuscript: all authors. Approved the final version of the manuscript on behalf of all authors: Whitaker-Lea.

\section{Correspondence}

Wittstatt Alexandra Whitaker-Lea: Virginia Commonwealth University Health System, Richmond, VA. wittstatt.whitakerlea@ vcuhealth.org. 